\title{
Space Telescope and Optical Reverberation Mapping Project: A Leap Forward in Reverberation Mapping
}

\author{
Bradley M. Peterson ${ }^{1,2,3}$ \\ ${ }^{1}$ Space Telescope Science Institute, 3700 San Martin Drive, Baltimore, MD 21218, USA \\ ${ }^{2}$ Department of Astronomy, The Ohio State University, 140 West 18th Ave., Columbus, OH \\ 43210, USA \\ ${ }^{3}$ Center for Cosmology and AstroParticle Physics, The Ohio State University, 191 West \\ Woodruff Ave., Columbus, OH 43210, USA \\ email: peterson.12@osu.edu
}

\begin{abstract}
In 2014, a 179-orbit allocation of Hubble Space Telescope time anchored a massive reverberation-mapping program on the well-studied Seyfert 1 galaxy NGC 5548. Supporting imaging and spectrophotometric observations were provided by Swift, Chandra, Spitzer, and a world-wide network of ground-based telescopes. Understanding the data remains a significant challenge, partly because the level of detail is far beyond what has been seen before and partly because the behavior of the AGN was not typical of its past behavior. Based on analysis to date, the following conclusions can be reached: (1) the AGN accretion disk has a temperature profile that is consistent with that predicted by the Shakura-Sunyaev model, but is about three times larger than expected; (2) at least part of the broad-line region appears to be a Keplerian disk seen at intermediate inclination, and (3) the broad-line emission response from the far side of the disk is weaker than expected.
\end{abstract}

Keywords. black hole physics, galaxies: active, galaxies: nuclei, quasars: emission lines, ultraviolet: galaxies

\section{Introduction}

Reverberation mapping is a technique for studying the structure and kinematics of the broad-line region (BLR) in AGNs by making use of the intrinsic flux variability of the central continuum source (Blandford \& McKee 1982, Peterson 1993, Peterson 2014). The relatively dense BLR gas responds to continuum variations with a time delay due to the light-travel time across the BLR. The amplitude of continuum and line flux variations is generally small on the relevant timescales, so a successful reverberationmapping campaign requires high $S / N$, high precision spectrophotometry, a high cadence, and a long duration (Horne et al. 2004) in order to recover velocity-delay maps, which are the projection of the BLR into the two observables of line-of-sight (Doppler) velocity and time delay (relative to the continuum variations). However, even with more modest data, it is possible to infer the mean BLR size simply by measuring the average response time for the entire emission line to the continuum variations - this is in fact what has been done in most reverberation studies for the past 25 years. Such measurements have been made for over 50 AGNs (see Bentz \& Katz 2015 for a compilation), and in some cases for multiple lines in a single AGN, but more often only for $\mathrm{H} \beta$.

The particular value of these measurements are that they can be combined with the width of the emission line to obtain a mass for the central black hole (see Peterson 2014 for details). In the case of AGNs for which multiple emission-line lag measurements 
have been made, the relationship between time lag $\tau$ and line width $\Delta V$ is found to be $\tau \propto \Delta V^{-1 / 2}$, which is as expected in system dominated by gravity. The mass of the central black hole is thus taken to be

$$
M_{\mathrm{BH}}=f\left(\frac{c \tau \Delta V^{2}}{G}\right),
$$

where $c$ is the speed of light, $G$ is the gravitational constant, and $f$ is a dimensionless scale factor of order unity that subsumes all of the details we do not know, such as the inclination of the system and its structure and kinematics. The factor $f$ is different for each AGN, but a statistical average value for AGNs can be inferred by assuming that AGNs follow the same relationship between black hole mass and host-galaxy bulge velocity dispersion as quiescent galaxies (Onken et al. 2004). Woo et al. (2015) provide the most recent update to this parameter, $\langle f\rangle=4.47 \pm 1.25$.

A key result from reverberation studies is the relationship between the BLR radius and the AGN luminosity, the $R-L$ relationship (Wandel, Peterson, \& Malkan 1999, Kaspi et al. 2000, Kaspi et al. 2005, Bentz et al. 2006, Bentz et al. 2009, Bentz et al. 2013). The great value of this relationship is that it allows us to bypass observationally expensive reverberation mapping by using a single spectrum to determine the AGN luminosity and the emission-line width to estimate the mass of the central black hole. All quasar mass estimates trace back to this relationship.

\section{Velocity-Resolved Reverberation Mapping}

While black-hole mass measurement has turned out to be an important byproduct of reverberation mapping, the actual goal remains characterization of the structure and kinematics of the BLR. After around a decade of experience with reverberation mapping, the continuum and emission line behaviors were sufficiently well characterized that we could address through simulations the question of what would it take to recover highfidelity velocity-delay maps (Horne et al. 2004). Even at the present time, velocitydependent lags have been identified for a limited number of objects (e.g., Bentz et al. 2009, Denney et al. 2009, Barth et al. 2011a, Barth et al. 2011b, Grier et al. 2013), and the number of AGNs for which velocity-delay maps have been recovered remains limited (Bentz et al. 2010, Grier et al. 2013). It must also be noted that a complementary approach of direct Bayesian modeling of the spectra has also shown to be very promising (Pancoast, Brewer, \& Treu 2011, Brewer et al. 2011, Li et al. 2013, Pancoast et al. 2014).

There is a perhaps interesting bit of sociology to note here: most reverberation results are based primarily on observations made on ground-based proprietary telescopes that are usually not the largest telescopes available to the observers. While reverberation mapping has been practiced in some form for over 25 years, it is a time-intensive risky $\dagger$ technique that is generally regarded as under development. As a consequence, most reverberation programs have been undertaken on smaller telescopes. The "reverberation sample" thus has several well-known biases compared to the AGN population as a whole: the reverberation-mapped AGNs tend to be lower-luminosity, apparently bright, high Eddington rate local AGNs that are primarily in the northern hemisphere. And space-based reverberation-mapping in the ultraviolet (UV) essentially ended with the decommissioning of the International Ultraviolet Explorer in 1996.

It is only when velocity-delay maps were successfully recovered from ground-based data (Bentz et al. 2010, Grier et al. 2013) that confidence in the reverberation-mapping

$\dagger$ Our experience with multiobject campaigns lasting months is that somewhat more than half of the targets will yield useful emission-line lags. 
technique was high enough that a proposal to carry out a UV reverberation program with Hubble Space Telescope (HST) was finally approved. The target selected for this study was NGC 5548, which is by far the best-studied of all the reverberation-mapped AGNs ("reverberated to death," in the words of one critic...) for a number of reasons, including that (1) its reverberation characteristics were of course well known, (2) historically it has little self-absorption in the UV resonance lines, and (3) it is bright enough to obtain a high $S / N$ spectrum in one $H S T$ orbit, and (4) its luminosity and line lags are almost optimal for efficient use of HST - daily observations for somewhat less than an entire observing season. The approved program consisted of 179 daily observations with the Cosmic Origins Spectrographł. The HST program was complemented by high-cadence observations with Swift and with ground-based imagining and spectroscopy, and we refer to the combined program as the AGN Space Telescope and Optical Reverberation Mapping (AGN STORM) program. The initial HST results are reported by De Rosa et al. (2015) and the Swift observations are described by Edelson et al. (2015). Fausnaugh et al. (2016) present the ground-based optical photometry and an analysis of the continuum behavior from the shortest $H S T$ band $(1158 \AA)$ to the Sloan $z$ band $(9157 \AA)$. Pei et al. (2016) present the results of the ground-based spectroscopy program, and Goad et al. (2016) and Starkey et al. (2016) describe some of the early analysis of the data.

The principal results to date include the following:

(1) The continuum variations at shorter wavelengths precede those at longer wavelengths. Throughout the UV to near-IR, the light curves at longer wavelengths look like shifted and smoothed versions of those at shorter wavelengths, as expected in simple lamp-post models (Edelson et al. 2015, Fausnaugh et al. 2016). The X-ray variations precede those in the UV-optical, but the X-ray light curves have more structure than those in the UV-optical - in other words, the UV-optical light curves are not shifted and smoothed versions of the X-ray light curves (Edelson et al. 2015, Gardner \& Done 2016).

(2) If the continuum variations are caused by irradiation of the accretion disk by a variable driving continuum, the variations can be used to map the accretion-disk structure. The disk temperature profile is consistent with the predictions of a standard ShakuraSunyaev thin disk, but the size of the disk is larger than expected by about a factor of three.

(3) The lag between the shortest-wavelength UV continuum and the optical continuum is approximately the same as the lag between the UV continuum and the most rapidly responding emission lines, He II $\lambda 1640$ and He II $\lambda 4686$ (De Rosa et al. 2015, Pei et al. 2016). This has important implications for optical-only reverberation programs.

(4) The emission-line behavior is not as simple as seen in previous reverberation observations. The responsivity of the emission lines varies during the campaign, which is unusual (Goad et al. 2016, Pei et al. 2016).

(5) Compared to previous reverberation campaigns on NGC 5548, the emission-line lags are unexpectedly small compared to expectations based on its luminosity. Based on luminosity, the $\mathrm{H} \beta$ lag, for example, should have been $\sim 20$ days, and the observed value is $\sim 6$ days (Pei et al. 2016). The equivalent width of $\mathrm{H} \beta$ is also surprisingly small, near its historically low value.

(6) The velocity-delay maps show that at least part of the BLR is a Keplerian disk viewed at intermediate inclination. However, the response of the far side of the BLR is weaker than expected (Horne et al., in preparation).

$\ddagger$ Due to safing events, only 171 of the observations were successfully executed, but the largest gap in the coverage is only two days. 
(7) The small lag, low equivalent width, and weak response of the far side of the BLR suggest that much of the ionizing radiation from the central source is not reaching the far side of the BLR, either because of absorption near the central source or anisotropic continuum emission.

(8) The resonance lines show both strong absorption in both broad and narrow components, which complicates the analysis. Historically, the absorption features have been weak in NGC 5548, but the situation has changed dramatically recently (Kaastra et al. 2014). We have carefully modeled the absorption in all the UV spectra in order to remove the absorption features. Further analysis based on these models is in progress (Kriss et al., in preparation).

We are grateful for support of this program by NASA through grant HST-GO-13330 from the Space Telescope Science Institute.

\section{References}

Barth, A. J., et al. 2011a, ApJ, 732:121

Barth, A. J., et al. 2011b, ApJ, 742, L4

Bentz, M. C. \& Katz, S. 2015, PASP, 127, 67

Bentz, M. C., et al. 2006, ApJ, 644, 133

-. 2009, ApJ, 697, 160

-. 2010, ApJ, 720, L46

-. 2013, ApJ, 767:149

Blandford, R. D. \& McKee, C. F. 1982, ApJ, 255, 419

Brewer, B. J., Treu, T., Pancoast, A., Barth, A. J., Bennert, V. N., Bentz, M. C., Filippenko, A. V., Greene, J. E., Malkan, M. A., \& Woo, J.-H. 2011, ApJ, 733, L33

De Rosa, G., et al. 2015, ApJ, 806:128

Denney, K. D., et al. 2009, ApJ, 704, L80

Edelson, R., et al. 2015, ApJ, 806:129

Fausnaugh, M. M., et al., ApJ, 821:56

Gardner, E. \& Done, C. 2016, MNRAS, in press

Goad, M. R., et al. 2016, ApJ, 824:11

Grier, C. J., et al. 2013, ApJ, 764:47

Horne, K., Peterson, B. M., Collier, S. J., \& Netzer, H. 2004, PASP, 116, 465

Kaastra, J., et al. 2014, Science, 345, 64

Kaspi, S., Maoz, D., Netzer, H., Peterson, B. M., Vestergaard, M., \& Jannuzi, B. T. 2005, ApJ, 629,61

Kaspi, S., Smith. P. S., Netzer, H., Maoz, D., Jannuzi, B. T., \& Giveon, U. 2000, ApJ, 533, 631

Li, Y.-R., Wang, J.-M., Ho, L. C., Du, P., \& Bai, J.-M. 2013, ApJ, 779:110

Onken, C. A., Ferrarese, L., Merritt, D., Peterson, B. M., Pogge, R. W., Vestergaard, M., \& Wandel, A. 2004, ApJ, 615, 645

Pancoast, A., Brewer, B. J., \& Treu, T. 2011, ApJ, 730: 139

Pancoast, A., Brewer, B. J., Treu, T., Park, D., Barth, A. J., Bentz, M. C., \& Woo, J.-H. 2014, MNRAS, 445, 3073

Pei, L., et al. 2016, submitted to ApJ

Peterson, B. M. 1993, PASP, 105, 247

-. 2014, Space Sci. Rev., 183, 253

Starkey, D., et al. 2016, submitted to ApJ

Wandel, A., Peterson, B. M., \& Malkan, M. A. 1999, ApJ, 526, 591

Woo, J.-H., et al. 2015, ApJ, 801:38 